\title{
PROBABILISTIC MODELING OF THE EFFICIENCY OF A STORMWATER DETENTION FACILITY
}

\author{
G. BECCIU \& A. RAIMONDI \\ Politecnico di Milano, Italy.
}

\section{ABSTRACT}

In modern urban drainage systems, stormwater detention facilities are important tools to reduce flood effects and discharges of uncontrolled pollutants into water receivers. For their design, simplified methods and continuous simulations of observed data are generally used. One of the main targets is to limit the frequency and the entity of overflows. In this article, a probabilistic approach for the derivation of the distribution functions of spilled volumes to evaluate the efficiency of a stormwater detention facility is proposed. The possibility of pre-filling of the storage capacity from a previous rainfall event, which can increase the probability of overflows from the stormwater detention facility, has been considered. The effects of the simplified assumptions have been deeply investigated and discussed. Final expressions have been applied to a case study, and results have been compared to those obtained from the continuous simulation.

Keywords: pre-filling, probabilistic approach, spills, stormwater detention facilities.

\section{INTRODUCTION}

In last decades, the growth of impervious surfaces combined with the ongoing climate changes has led to a significant increase of floods. These cause considerable damages especially in urban areas, where the drainage system is often insufficient to convey large runoff volumes.

Stormwater detention facilities are often used to store rainfall volumes, which exceed the capacity of the existing network, reducing flood effects. Moreover, they have the environmental benefit of avoiding uncontrolled discharges of pollutants into receiving waters [1,2].

In their design, one of the main objectives is to limit spilled volumes, increasing the efficiency of the facility. Often, for the estimation of the storage capacity simplified approaches are used. One of the most popular, the so-called 'design storm' approach, is based on a 'critical' rainfall event, extracted from a recorded series of rainfall data or defined by a standard pattern (rectangular, triangular, Chicago, etc.), as input in a rainfall-runoff model [3]. This method assumes the 'critical' event isolated from the stochastic process of rainfalls, considering the storage volume always empty at the beginning of each runoff. The possibility of pre-filling of the storage capacity from previous rainfall events is neglected so that an underestimation of the minimum storage volume can occur.

To consider the whole chain of runoff events, continuous simulation of observed rainfalls is often suggested. It allows obtaining more reliable and accurate results, but its use is conditioned to the availability of long-term series of records $[4,5]$.

To overcome the limitations of both simplified approaches and continuous simulations, in 1990s, some authors [6-9] developed an analytical probabilistic approach for the modelling of stormwater detention facilities. It allows deriving the probability distribution functions of some characteristics of the storage process from those of the rainfall variables and from the characteristics of the drainage catchment. Resulting expressions are generally easy to implement and reliable from a probabilistic point of view. In recent years, many applications and developments of this kind of approaches have been proposed [10-13].

In particular, the authors of [14-16] have deeply investigated the problem of pre-filling of the storage capacity from previous rainfall events, often neglected or just mentioned in the literature. 
Pre-filling volumes from previous rainfall events highly increase the risk of spills from the storage capacity, reducing the environmental and flood protection benefits of the facility.

This article focuses on the analytical probabilistic derivation of the distribution functions of spilled volumes, important to evaluate the efficiency of a stormwater detention facility.

The possibility of pre-filling from a previous rainfall event is considered and the effects of simplifying hypothesis, made in the schematization of the process, on results have been deeply investigated and discussed.

Finally, derived formulas from the proposed approach have been tested by their application to a case study in Milano, Italy, and results have been compared to those obtained from the continuous simulation of observed rainfalls data.

\section{MODELING OF THE STORAGE PROCESS}

The filling-emptying process from a stormwater detention facility has been schematized by means of some simplifying assumptions described below:

- Independent rainfall events have been identified from the continuous series of records. To this aim, a minimum intervent time IETD between two consecutive rainfall events has been defined; if the interevent time $d$ is lower than IETD, two consecutive rainfalls have been joined together into a single event, otherwise they have been considered independent.

- The main characteristics of the rainfall process: rainfall depth $h$, rainfall duration $\theta$ and interevent time $d$, have been considered as independent random variables exponential distributed:

$$
\begin{gathered}
f_{h}=\xi \cdot e^{-\xi \cdot h} \\
f_{\theta}=\lambda \cdot e^{-\lambda \cdot \theta} \\
f_{d}=\psi \cdot e^{-\psi \cdot(d-I E T D)}
\end{gathered}
$$

where: $\xi=1 / \mu_{h}, \lambda=1 / \mu_{\theta}$ and $\psi=1 /\left(\mu_{d}-I E T D\right)$, with $\mu_{h}$ the average rainfall depth, $\mu_{\theta}$ the average rainfall duration and $\mu_{d}$ the average interevent time.

- On-line stormwater detention facilities have been considered: when the storage capacity is full and inflow occurs at a greater rate than outflow, the excess volume is spilled.

- A couple of events, isolated from the stochastics process of rainfalls, has been considered, that is, it has been assumed that the storage volume can be pre-filled from no more than one previous rainfall event. As discussed by Raimondi and Becciu [16], this hypothesis can be reliable only when a sufficiently long InterEvent Time Definition IETD and high outflow rates are considered. For stormwater detention facilities with low outflow rates (e.g. infiltration basins) or when strict limitations on discharges in the downstream drainage system are imposed, this hypothesis can underestimate the pre-filling probability and, as consequence, the overflow probability.

- Incoming hydrographs have been assumed rectangular, that is, constant runoff has been considered; this hypothesis can be acceptable since in the modelling of stormwater detention facilities, rainfall volumes are more influent than rainfall intensities. Moreover, the pattern of incoming hydrographs can be neglected, especially when inflow rates are higher than outflow rates.

- Hydrological losses $L$ for infiltration, evapotranspiration and filling of depression storages have been averaged over the whole rainfall duration, that is, they are constant and do not vary with rainfall intensity. 


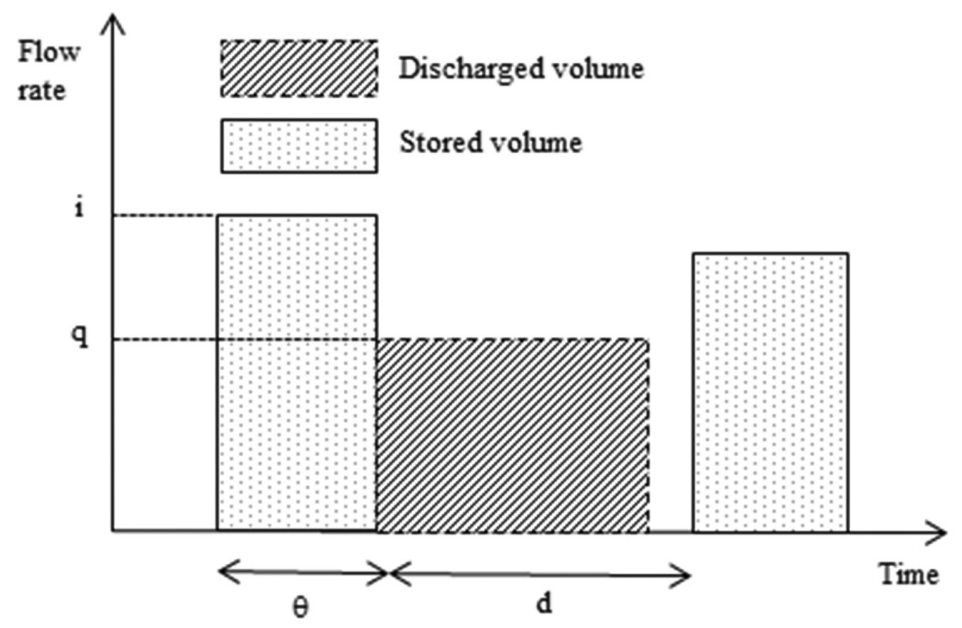

Figure 1: Schematization of the filling-emptying process from a stormwater detention facility.

- Rainfall-runoff transformation has been neglected and net rainfall intensities $i$ have been considered as inflow rates in the basin. This hypothesis can be reliable only for small catchments; as a consequence, runoff duration is considered equal to rainfall duration, although the duration of a runoff event is usually longer than that of a rainfall event.

- The stormwater detention facility is emptied, with a constant outflow rate $q$, only after the end of each runoff event (Fig. 1). The outflow goes on until the facility is empty or a next event begins. This management rule is often used in real-time control applications, when it is necessary to temporarily retain a certain volume to reduce the risk of downstream system overload, or for water quality control purposes.

\section{PROBABILISTIC ANALYSIS OF SPILLS}

The efficiency of a stormwater detention facility can be evaluated by assessing its capacity to cope with spills. With reference to a couple of consecutive rainfall events (Fig. 1), the conditions of spills are:

- spill of a single event only;

- spill of both runoff events with pre-filling from the first event at the beginning of the second one; and

- spill of the second runoff event only, with pre-filling from the first event at the beginning of the second one.

In particular, the probability that the spilled volume $w_{s}$ exceeds a percentage $a$ of the storage capacity $w_{0}$ has been calculated, that is, the probability $\operatorname{Prob}\left(w_{s}>a \cdot w_{0}\right)$.

Two different conditions have been distinguished in the probabilistic derivation. The possibility that spilled volume is influenced by pre-filling from the previous rainfall event, defined by the condition $w_{0} / q>I E T D$ and the hypothesis that spilled volume is only due to a single rainfall event, defined by the condition $w_{0} / q \leq I E T D . w_{0} / q$ is the time that the storage capacity $w_{0}$, completely 
filled, takes to empty with a constant outflow $q$. If it results less than IETD, the minimum interevent time, isolated rainfall events have been considered.

In all expressions, volumes and flow rates have been expressed per unit of effective catchment area $\varphi \cdot S$, where $\varphi$ is the runoff coefficient and $S$ is the catchment area. Furthermore, the following auxiliary dimensionless variables have been used: $\beta=q \cdot \xi /(q \cdot \xi+2 \cdot \psi)$ and $q^{*}=q \cdot \xi / \lambda$.

If the stormwater detention facility is emptied, with a constant outflow rate $q$, only after the end of each runoff event (Fig. 1), and the possibility of spill from a single event only is considered, that is, condition $w_{0} / q \leq I E T D$ is assumed, spilled volume $w_{s}$ can be expressed by:

$$
w_{s}=h-L-w_{0} \quad \text { case } I .
$$

where case I has the condition:

$h-L-w_{0}>0$;

Introducing in eqn (4) the probability distribution function of rainfall depth, eqn (1), it becomes:

$$
P=\operatorname{Prob}\left(w_{s}>a \cdot w_{0}\right)=\int_{h_{A}}^{h_{B}} f_{h} \cdot d h=e^{-\xi\left[L+w_{0} \cdot(1+a)\right]}
$$

where:

$h_{A}=L+w_{0} \cdot(1+\alpha) ; h_{B}=\infty$.

If the possibility of pre-filling from the previous rainfall event is considered, that is, condition $w_{0} / q>$ IETD is assumed, spilled volume $w_{s}$ results:

$$
w_{s}= \begin{cases}h-L-w_{0} & \text { case I. } \\ \sum_{i=1}^{2} h_{i}-2 \cdot L-q \cdot d-w_{0} & \text { case II, case III. } \\ 0 & \text { otherwise }\end{cases}
$$

where case I has the condition:

$h-L-w_{0}>0$.

case II has the conditions:

$h-L-w_{0}>0$;

$w_{0}-q \cdot d>0$;

$\sum_{i=1}^{2} h_{i}-2 \cdot L-q \cdot d-w_{0}>0$.

and case III has the conditions:

$h-L-w_{0} \leq 0$;

$h-L-q \cdot d>0$;

$\sum_{i=1}^{2} h_{i}-2 \cdot L-q \cdot d-w_{0}>0$. Last condition in case II and case III, $\sum_{i=1}^{2} h_{i}-2 \cdot L-q \cdot d-w_{0}>0$, can be expressed by means of
the simplified form: 
(a) $2(h-L)-q \cdot d-w_{0}>0$

or by

(b) $h^{*}-2 \cdot L-q \cdot d-w_{0}>0$

where $h^{*}=\sum_{i=1}^{2} h_{i}$ has a Gamma probability distribution function.

Condition (a) assumes, as simplifying hypothesis, that the sum of two exponential probability distribution functions is an exponential probability distribution function too. Condition (b) instead considers the sum of two exponential probability distribution functions as Gamma distributed. The purpose of testing the two different conditions (a) and (b) in the derivation of the probability distribution function of spilled volumes is to verify the goodness of the simplifying hypothesis (a), that is generally simpler to apply in practice.

Considering expression (a), (eqn 7), the definition of spilled volume $w_{s}$ (eqn 6) and the probability distribution functions of rainfall depth and interevent time (eqns 1 and 3, respectively), the probability distribution function of spilled volumes results:

$$
\begin{aligned}
& P_{a}=\operatorname{Prob}\left(w_{s}>\alpha \cdot w_{0}\right)=\int_{d_{A}}^{d_{B}} f_{d} \cdot d d \cdot \int_{h_{A}}^{h_{B}} f_{h} \cdot d h+\int_{h_{C}}^{h_{D}} f_{h} \cdot d h \\
& =(1-\beta) \cdot e^{-\xi \cdot L} \cdot\left\{e^{-\frac{\xi}{2} \cdot\left[\cdot\left[\cdot I E T D+w_{0} \cdot(1+\alpha)\right]\right.}-e^{\psi \cdot \operatorname{lETD}-w_{0} \cdot\left[\xi+\frac{\psi}{q} \cdot(1-\alpha)\right]}\right\}+e^{-\xi \cdot\left[L+w_{0} \cdot(1+\alpha)\right]}
\end{aligned}
$$

where:

$$
\begin{aligned}
& d_{A}=I E T D ; d_{B}=w_{0} \cdot(1-a) / q, h_{A}=L+\left[w_{0} \cdot(1+a)+q \cdot d\right] / 2 ; h_{B}=L+w_{0} \text { and } \\
& h_{C}=L+w_{0} \cdot(1+a) ; h_{D}=\infty .
\end{aligned}
$$

Considering expression (b) (eqn 8), and the definition of spilled volume $w_{s}$ (eqn 6), the probability distribution function of spilled volumes can be expressed by:

$$
P_{b}=\operatorname{Prob}\left(w_{s}>\alpha \cdot w_{0}\right)=\int_{d_{A}}^{d_{B}} f_{d} \cdot d d \cdot \int_{h_{A}^{*}}^{h_{B}^{*}} f_{h^{*}} \cdot d h^{*}+\int_{h_{A}}^{h_{B}} f_{h} \cdot d h
$$

where $d_{A}=I E T D ; d_{B}=w_{0}(1-a) / q, h_{A}^{*}=w_{0} \cdot(1+a)+q \cdot d+2 \cdot L ; h_{B}^{*}=\infty$ and $h_{A}=L+w_{0}$. $(1+a) ; h_{B}=\infty$.

By substituting eqns (1), (3) and (8) in eqn (10), it results

$$
\begin{aligned}
& P_{b}=\frac{\delta \cdot \psi}{\left(\mu_{h}-2 \cdot L\right)^{2}} \cdot e^{\psi \cdot I E T D-\frac{w_{0} \cdot(1+\alpha)}{\mu_{h}-2 \cdot L}} \cdot\left[1+w_{0} \cdot(1+\alpha)\right] . \\
& \left\{e^{-\frac{I E T D}{\delta}} \cdot[1+q \cdot(I E T D+\delta)]-e^{-\frac{w_{0} \cdot(1-\alpha)}{\delta \cdot q}} \cdot\left[1+w_{0} \cdot(1-\alpha)+\delta \cdot q\right]\right\}+e^{-\xi \cdot\left[L+w_{0} \cdot(1+\alpha)\right]}
\end{aligned}
$$


where $\delta=\frac{\mu_{h}-2 \cdot L}{\psi \cdot\left(\mu_{h}-2 \cdot L\right)+q}$.

Threshold conditions of the probability distribution functions of spilled volumes, eqns (5), (9) and (11), are:

$$
\begin{gathered}
\lim _{w_{0} \rightarrow 0} P=e^{-\xi \cdot L} \\
\lim _{w_{0} \rightarrow \infty} P_{a}=\lim _{w_{0} \rightarrow \infty} P_{b}=0 \\
\lim _{q \rightarrow \infty} P=0 \\
\lim _{q \rightarrow 0} P_{a}=e^{-\xi \cdot\left[L+w_{0} \cdot(1+\alpha)\right]} \\
\lim _{q \rightarrow 0} P_{b}=\frac{1}{\left(\mu_{h}-2 \cdot L\right)^{2}} \cdot e^{-\frac{w_{0} \cdot(1+\alpha)}{\left(\mu_{h}-2 \cdot L\right)}} \cdot\left[1+w_{0} \cdot(1+\alpha)\right]+e^{-\xi \cdot\left[L+w_{0} \cdot(1+\alpha)\right]}
\end{gathered}
$$

\section{APPLICATION}

Derived expressions for the estimation of the probability distribution functions of spilled volumes have been validated by their application to the rainfall series recorded at gauge station of MilanoMonviso (Italy) during the period 1991-2005. An IETD = $10 \mathrm{~h}$ has been used in the analysis, corresponding to the identification of 979 rainfall events from the continuous chain of records. Average values of rainfall depth, rainfall duration and interevent time are shown in Table 1, while standard deviation and variation coefficients are shown, respectively, in Tables 2 and 3.

Table 1: Average values of rainfall characteristics.

\begin{tabular}{lll}
\hline$\left.\mu_{h}(\mathrm{~mm})\right]$ & $\mu_{\vartheta}(\mathrm{h})$ & $\mu_{d}(\mathrm{~h})$ \\
\hline 18.49 & 14.37 & 172.81 \\
\hline
\end{tabular}

Table 2: Standard deviation of rainfall characteristics.

\begin{tabular}{lll}
\hline$\sigma_{h}(\mathrm{~mm})$ & $\sigma_{\vartheta}(\mathrm{h})$ & $\sigma_{d}(\mathrm{~h})$ \\
\hline 21.33 & 14.81 & 223.89 \\
\hline
\end{tabular}

Table 3: Variation coefficients of rainfall characteristics.

\begin{tabular}{lcc}
\hline$V_{h}$ (dimensionless) & $V_{\theta}$ (dimensionless $)$ & $V_{d}$ (dimensionless $)$ \\
\hline 1.15 & 1.03 & 1.30 \\
\hline
\end{tabular}


Resulting values of variation coefficients, shown in Table 3, point out that only rainfall duration well fits an exponential distribution function (coefficient of variation $V \cong 1$ ). This aspect was previously discussed by Guo and Adams [7] and Raimondi and Becciu [16] that concluded that for most of Italian basins, the Weibull and the double-exponential probability distribution functions are more correct to fit experimental data. Anyway, the gain in results accuracy from the use of such probability distribution functions is minimal compared to the increasing complexity in derivation of probabilistic expressions. The use of an exponential distribution function to fit series of rainfall data is widespread in the literature to reduce the complexity of analytical derivation [17-22]. Correlation coefficients among rainfall variables are shown in Table 4:

While correlation between rainfall depth and interevent time and rainfall duration and interevent time is quite low, there is a strong correlation between rainfall depth and rainfall duration. As discussed by Becciu and Raimondi [23], this can lead to a deviation of results of derived formulas from those from the continuous simulation. However, since the assumption that the stormwater detention facility is emptied only after the end of each runoff event has been considered, the dependence of the probability distribution function of spilled volumes from rainfall duration is excluded, and the correlation between rainfall depth and duration does not influence the results.

In the application, hydrological losses $L$ have been considered equal to $2 \mathrm{~mm}$ and the constant outflow rate $q$ has been assumed equal to $1 \mathrm{~mm} / \mathrm{h}$, less than the average rainfall intensity $\mu_{i}=\mu_{h} / \mu_{\vartheta}$, equal to $1.29 \mathrm{~mm} / \mathrm{h}$.

Results from derived formulas have been compared to results from the continuous simulation of observed data, schematization of which is shown in Fig. 2:

Table 4: Correlation coefficients among rainfall characteristics.

\begin{tabular}{ccc}
$\rho_{h, \theta}$ & $\rho_{h, d}$ & $\rho_{d, \theta}$ \\
\hline 0.62 & 0.11 & 0.11
\end{tabular}

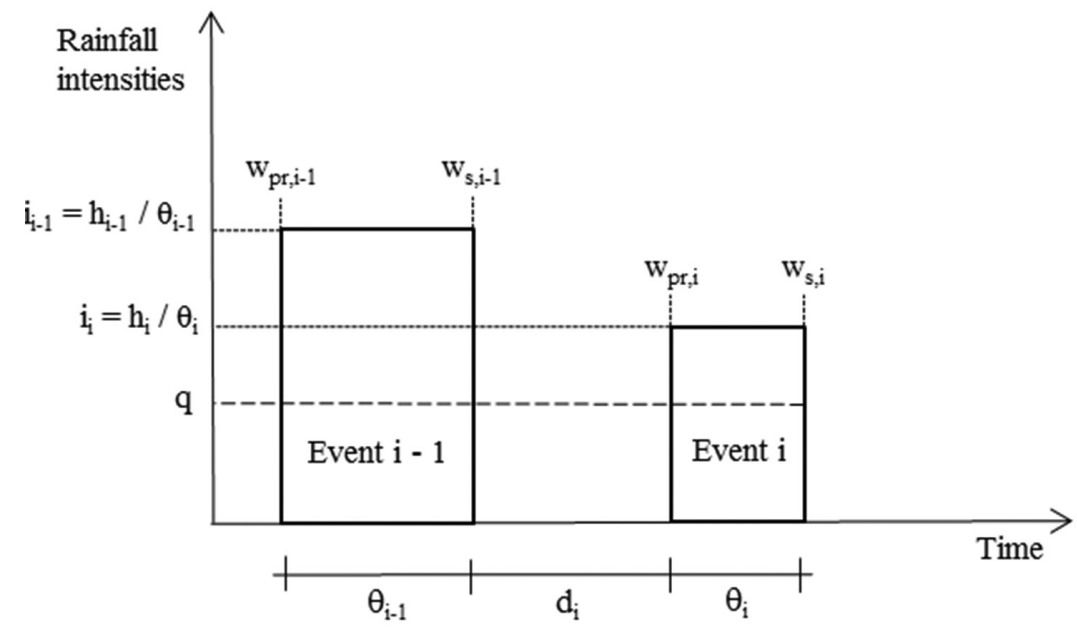

Figure 2: Schematization of two consecutive runoff events. 
Reference equations are:

$$
w_{p r, i}= \begin{cases}w_{p r, i-1}+h_{i-1}-L-q \cdot d_{i} & \text { case I } \\ w_{p r, i-1}+w_{0}-q \cdot d_{i} & \text { case II } \\ 0 & \text { otherwise }\end{cases}
$$

$w_{p r, i}$ is the pre-filling volume at the beginning of the rainfall event $i$ and $w_{p r i-i}$ is the pre-filling volume at the beginning of the rainfall event $i-1$.

case I represents the conditions:

$h_{i-1}-L-w_{0} \leq 0$;

$h_{i-1}-L-q \cdot d_{i}>0$.

and case II represent the conditions:

$h_{i-1}-L-w_{0}>0$;

$w_{0}-q \cdot d_{i}>0$.

$$
w_{s, i}= \begin{cases}w_{s, i-1}+w_{p r, i}+h_{i}-L-w_{0} & \text { case I } \\ w_{p r, i-1}+h_{i}-L-w_{0} & \text { case II } \\ 0 & \text { otherwise }\end{cases}
$$

$w_{s, i}$ is the spilled volume at the end of the rainfall event $i$ and $w_{s, i-i}$ is the spilled volume at the end of the rainfall event $i-1$.

where case I represents the conditions:

$w_{p r, i}>0$

$w_{s, i-1}+w_{p r i}+h_{i}-L-w_{0}>0$;

and case II represent conditions:

$w_{p r, i} \leq 0$

$h_{i}-L-w_{0}>0$.

Figure 3 shows the probability distribution function of spilled volumes, comparing results from continuous simulation and derived formulas for $\alpha=0$, that is, the probability of spilled volumes different from 0 .

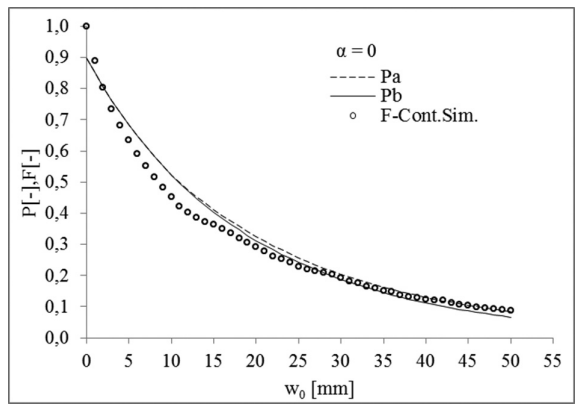

Figure 3: Overflow probability versus storage volume for $\alpha=0$. 


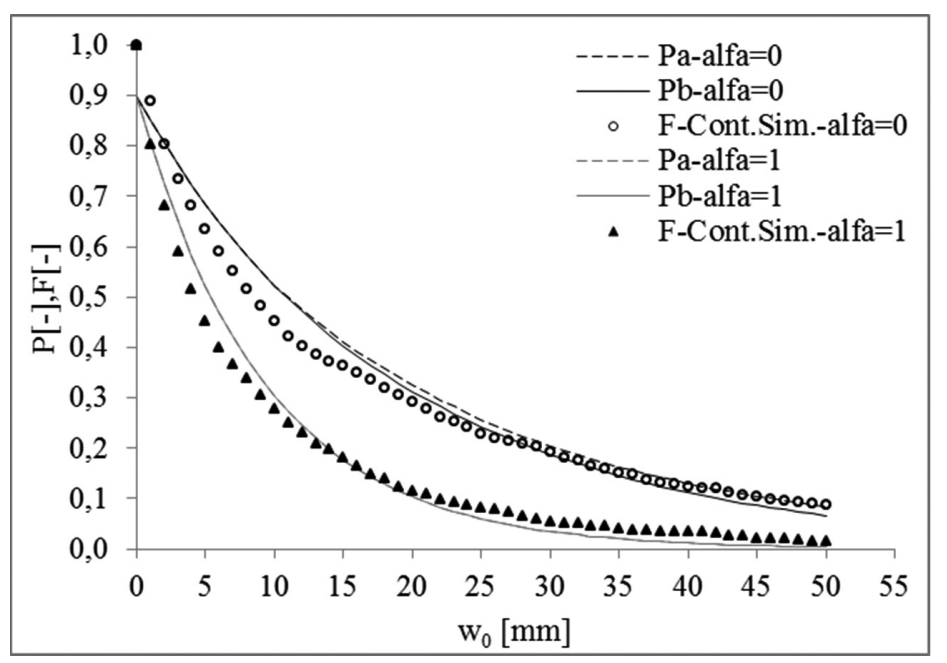

Figure 4: Probability distribution functions of spills versus storage volume for $a=0$ and $a=1$.

The use of the simplified expression (a) in the derivation of the probability distribution function of spilled volumes involves negligible differences in results with respect to complete expression (b) and can be easily applied in practice.

Figure 4 compares the probability distribution functions of spilled volumes for the two threshold conditions of the $a$ coefficient, that is, the probability of spilled volumes higher than $0, \operatorname{Prob}\left(w_{s}>0\right)$, with $a=0$, and the probability of spilled volumes higher than the storage capacity, $\operatorname{Prob}\left(w_{s}>w_{0}\right)$, with $\alpha=1$.

Obviously, with $a=0$ the probability distribution function of spilled volumes is higher than for $a=1$. In this case, the use of a simplified expression (a) is equivalent to complete expression (b).

Differences between results from the application of derived formulas and the continuous simulation of observed data can be mainly due to the simplified assumptions made in the derivation: the hypothesis of independence of the rainfall characteristics, the hypothesis of considering only a couple of rainfall events at time and/or the hypothesis of exponential distribution of rainfall characteristics.

In particular, results from the application of the final expressions are little affected from the simplifying assumption of independence of rainfall variables, except for little correlation between rainfall depth and interevent time (see Table 4).

Moreover, as discussed by Raimondi and Becciu [16], with an IETD $=10 \mathrm{~h}$, the hypothesis of considering only a couple of rainfall events a time can be acceptable, since only in few cases the pre-filling of the storage capacity is due to more than on previous rainfall event. This assumption influences more the probability distribution function of spilled volumes for $\alpha=0$, as shown in Fig. 4 .

The effects of the hypothesis of the exponential distribution of rainfall variables could be tested by the use of a Weibull or a double-exponential distribution function that best fit the frequency distribution of rainfall depth and interevent time $[7,16]$.

\section{CONCLUSIONS}

The efficiency of stormwater detention facilities is very important for environmental purposes and flood protection of downstream areas. The reduction of spills from these facilities should be one of the main targets in their design. 
In this article, an innovative approach for the estimation of the efficiency of stormwater detention facilities to cope with overflows has been discussed. It is based on the analytical probabilistic derivation of the distribution functions of spilled volumes. With reference to a couple of rainfall events, the proposed method allows considering the effects of the pre-filling from the first runoff on the spilled volume at the end of the second one.

Derived expressions are very simple to apply and can be a valuable support to engineers in the design of stormwater detention facilities. The probability distribution functions of spilled volumes result mainly function of the average values of the rainfall characteristics, storage capacity and outflow rate. They also allow evaluating the probability to have a certain amount of spilled volume in relation to the storage capacity. Moreover, final expressions take into account the minimum interevent time used for the identification of independent rainfall events and the hydrological losses for infiltration, evapotranspiration and filling of depression storages.

Effects of all simplifying hypothesis on resulting formulas have been deeply investigated and discussed. The reliability of the proposed approach has been tested by its application to a case study. Goodness of derived formulas has been confirmed by the comparison between results from their application with those from the continuous simulation of observed data. The use of the proposed approach can be suitable in many engineering applications, especially when strict discharge limits increase the probability of spills from stormwater detention facilities and when the continuous simulation of series of recorded storm events is not possible or reliable according to the amount and quality of rainfall records.

\section{REFERENCES}

[1] Urbonas, B. \& Stahre, P., Stormwater: Best Management Practices and Detention for Water Quality, Drainage and CSO Management, PTR Prentice Hall: Englewood Cliffs, NJ, 1993.

[2] ASCE \& WEF, Urban Runoff Quality Management, American Society of Civil Engineers: Reston, VA, 1998.

[3] Dooge, J.C.I., Problems and methods of rainfall runoff modeling, Mathematical Models for Surface Water Hydrology, eds. T.A. Ciriani, U. Maione, \& J.R. Wallis, John Wiley: NY, pp. 71-108, 1977.

[4] Adams, B.J., Fraser, H.G., Howard C.D.D. \& Hanafy, M.S., Meteorologic data analysis for drainage system design. Journal of Environmental Engineering, 112(5), pp. 827-848, 1986. doi: http://dx.doi.org/10.1061/(asce)0733-9372(1986)112:5(827)

[5] Bacchi, B., Balistrocchi, M. \& Grossi G., Proposal of a semi - probabilistic approach for storage facility design. Urban Water Journal, 5(3), pp. 195-208, 2008. doi: http://dx.doi. org/10.1080/15730620801980723

[6] Guo, Y. \& Adams, B.J., Hydrologic analysis of urban catchments with event-based probabilistic models. 1. Runoff volume. Water Resources Research, 34(12), pp. 3421-3431, 1998. doi: http://dx.doi.org/10.1029/98wr02449

[7] Guo, Y. \& Adams, B.J., Hydrologic analysis of urban catchments with event-based probabilistic models. 2. Peak discharge rate. Water Resources Research, 34(12), pp. 3433-3443, 1998. doi: http://dx.doi.org/10.1029/98wr02448

[8] Guo, Y. \& Adams, B.J., An analytical probabilistic approach to sizing flood control detention facilities. Water Resources Research, 53(8), pp. 2457-2468, 1999. doi: http://dx.doi. org/10.1029/1999wr900125

[9] Adams, B.J. \& Papa, F., Urban Stormwater Management Planning With Analytical Probabilistic Models, John Wiley \& Sons: New York, 1999. doi: http://dx.doi.org/10.1016/ s1462-0758(01)00014-0 
[10] Guo, Y. \& Dai, J., Expanded analytical probabilistic stormwater models for use in watershed and master drainage planning. Canadian Journal of Civil Engineering, 36(6), pp. 933-943, 2009. doi: http://dx.doi.org/10.1139/109-037

[11] Guo, Y. \& Zhuge, Z., Analytical probabilistic flood routing for urban stormwater management purposes. Canadian Journal of Civil Engineering, 35(5), pp. 487-499, 2008. doi: http://dx.doi. org/10.1139/107-131

[12] Raimondi, A. \& Becciu, G., Probabilistic modeling of rainwater tanks. Procedia Engineering, 89, pp. 1493-1499, 2014. DOI: 10.1016/j.proeng.2014.11.437. doi: http://dx.doi.org/10.1016/j. proeng.2014.11.437

[13] Raimondi, A. \& Becciu, G., Probabilistic design of multi-use rainwater tanks. Procedia Engineering, 70, pp. 1391-1400, 2014. doi: http://dx.doi.org/10.1016/j.proeng.2014.02.154

[14] Becciu, G., Paoletti, A., Raimondi, A. \& Sanfilippo U., Effects of management rules on pre-filling of stormwater detention facilities. Proceedings of 12th International Conference on Urban Drainage, Porto Alegre, Brazil, 2011.

[15] Becciu, G. \& Raimondi, A., On pre-filling probability of storage tanks. WIT Transactions: Ecology and the Environment, 164 (Water Pollution XI), 2012.

[16] Raimondi, A. \& Becciu, G., On pre-filling probability of flood control detention facilities, Urban Water Journal, 12(4), pp. 344-351, 2015. doi: 10.1080/1573062X.2014.901398.

[17] Eagleson, P.S., Dynamics of flood frequency. Water Resources Research, 8(4), pp. 878-898, 1972. doi: http://dx.doi.org/10.1029/wr008i004p00878

[18] Eagleson, P.S., Climate, soil and vegetation, 2. The distribution of annual precipitation derived. Water Resources Research, 14(5), pp. 713-721, 1978. doi: http://dx.doi.org/10.1029/ wr014i005p00713

[19] Howard, C.D.D., Theory of storage and treatment plant overflows. Journal of Environmental Engineering, 102(EE4), pp. 709-722, 1976.

[20] Adams, B.J. \& Bontje, J.B., Microcomputer applications of analytical models for urban stormwater management. Emerging Computer Techniques in Stormwater and Flood Management, ed. W. James, American Society of Civil Engineers: New York, pp. 138-162, 1984.

[21] Adams, B.J., Fraser, H.G., Howard C.D.D. \& Hanafy, M.S., Meteorologic data analysis for drainage system design. Journal of Environmental Engineering, 112(5), pp. 827-848, 1986. doi: doi: http://dx.doi.org/10.1061/(asce)0733-9372(1986)112:5(827)

[22] Bedient, P.B. \& Huber, W.C., Hydrology and floodplain analysis, 2nd edn, Addison Wesley: New York, 1992.

[23] Becciu, G. \& Raimondi, A., Probabilistic analysis of spills from stormwater detention facilities, WIT Transactions on the Built Environment, 139, 2014, doi:10.2495/UW140141. doi: http:// dx.doi.org/10.2495/uw140141 